\section{SOME FURTHER NOTES ON THE USE OF BROMIDE OF STRONTIUM IN EPILEPSY.}

BY ANTONY ROCHE, M.R.C.P. IREL.,

PROFESSOR OF MKDICAT JURISPRUDENCE AND PUBLIC HEAL'TH IN TH CATHOLIC UNIVERSITY MEDICAL SCHOOL, DUBLIN ; EXAMINER TO THE ROYAL UNIVERSITY OF IRELAND.

IN 1894, and again in 1896, I published some notes, with cases, on the use of the bromide of strontium in epilepsy. I have many of the cases then mentioned still under my observation, with a considerable number of others. Perhaps therefore some remarks founded on a more extended experience in the use of this bromide may be of interest to the profession. Unfortunately I cannot give the results in all my cases, as some of my patients communicated with me only by letter, and I am therefore unable to follow the course in all. In the majority of cases, however, I have been able to learn the results of the treatment.

I have not met any case in which the bromide of strontium given in the doses and method I will mention has failed to diminish the number of the attacks. In many instances there has been no return for periods extending to two, three, and even four years. Some of these cases may be considered "cured"-that is, that there will be no return of the attacks when the medicine is permanently left off. I cannot say definitely that this is so, for the reason that $I$ always recommend the patients to continue the medicine, even though there has been no return for a long period. I do this all the more as I have not found the continued use of bromide of strontium to be followed by any bad consequences, and many of the patients have been taking 1 drachm daily without intermission for more than three years without any complaint. In this respect the strontium salt has an immense advantage over the potassium salt, which has frequently produced serious physical and mental changes when continued for long periods. Moreover, patients taking bromide of potassium frequently suffer from intense depression. Several of my patients were obliged to leave it off owing to this ; in fact, several expressed their feelings by assuring me that they would rather suffer from the fits than from the depression produced by the medicine. I have not noticed this at all in those using the bromide of strontium. The potassium, too, is poisonous in large doses; the strontium is not. On this point I may quote one of the most recent authorities, Dr. Dixon Mann, who in the second edition of his excellent "Manual of Medical Jurisprudence" says that "strontium salts cannot be regarded as poisonous." I have myself given 3 drachms daily for weeks without any unpleasant symptoms. I consider, therefore, that the strontium salt excels that of potassium in being more efficient and in not being followed by dangerous consequences when it is continued over a lengthened period; and as it is not poisonous it can be given in as large a dose as may be required to control the attacks.

In my earlier cases, being influenced by the remark of Brown-Sequard that the combined bromides were more useful, I gave the bromide of strontium combined with one or more of the other bromides, but for a long time I have given that of strontium alone. I usually commence the treatment by ordering half a drachm night and morning in some vegetable tonic infusion. Should that dose not control the attacks I rapidly increase it till I have found the quantity which will suit the individual case. I direct the patient to take 30 grains at once in those cases where there is any warning of the attack and to repeat this every hour if required. By this means I have no doubt the attack has been frequently prevented. I may repeat that in my experience in order to get the full benefit of the medicine I have found it necessary to give it in large doses and to continue it for a long period. Since my first communication several members of the profession have written to me saying that in their cases they have not found the same good results. I invariably find that in these cases the dose given has been too small and consequently that my practice has not been followed. I need not say that each case requires to be studied on its own merits and any exciting or predisposing cause lessened or removed. I do not think it necessary to go any further into this question, as these notes are not upon the general medical or surgical treatment of epilepsy, but upon a special medicine, nor need I give my experience in the use of any other of the many remedies proposed. Many of these I have tried, but none have given me the same good results as I have found by the use of bromide of strontium.

I should say, however, as I have more fully explained on a former occasion, that $I$ regard the question of diet as one of much importance. I direct my patients to restrict themselves to fish and vegetables, and I generally find no grtat difficulty in having my orders carried out. When the attacks diminish in number, if I am pressed to do so I allow the more easily digested meats twice or three times in the week.

I propose to return to this subject in a subsequent communication and to give details of some of my cases. Dullin.

\section{THE $\mathrm{X}$ RAYS IN DISEASES OF THE CHEST.}

By HUGH WALShaM, M.A., M.D. Cantab., M.R.C.P. LOND.,

ASSISTANT PHYSICIAN TO THE CITY OF LONDON HOSPITAT TOF DISEASES OF THE CHEST; ASSIS'TAY'T MEDICAL OFFICER IN THE ELECTRICAL DEPARTMEXT, S'I. BARTHOLONEW'S HOSPITAL; ATI) CLINICAL ASSISTAN'T TO THE HOSPITAL FOR SICK CHILDREN, GREAT ORMOND-STREET.

THE experience of the last three years has been quite sufficient to prove beyond question the usefulness of Professor Roentgen's discovery to the surgeon. I venture to think, however, that a systematic application of the rays to the diagnosis of chest disease will prove of equal value to the physician. From some cause the $x$ rays have up to the present time not been so extensively used by the physician as they have by the surgeon, and yet they are capable of showing very distinctly pathological conditions within the chest. Unfortunately at present we have not the apparatus necessary at the City of London Hospital for Diseases of the Chest to examine patients ; but what little I have been able to do thoroughly convinces me of their great value in chest disease, more especially in diseases of the lung. This, I think, will be quite apparent from an examination of the two accompanying skiagrams.

The first (Fig. 1) is from the chest of a perfectly healthy boy, aged nine years. It is seen at once that both lungs in $a_{x}$ healthy condition are perfectly transparent to the rays from apex to base, allowing the posterior portions of the ribs to be clearly seen. Some slight shading will be seen on each side corresponding to the position of the larger bronchi, but with this exception the healthy lung is quite transparent to the rays. Whether this transparency continues with advancing life I am not quite sure, probably it does not. I think that, as life advances the lung becomes somewhat less transparent, but this of course affects the whole lung equally. If we now turn to Fig. 2 we see at once that there is a great. difference. The skiagram is from the chest of a boy, aged fifteen years, who came under my care when was in charge of Dr. Harrington Sainsbury's wards during the past summer. Physical examination of the chest gave signs of a carity at both apices. On the left side. the physical signs of the cavity extended from the apex downwards to the level of the third rib. I was therefore much surprised on examining this patient with the fluorescent screen to find that the cavity was, comparatively speaking, a small one, certainly not extending so far down.wards as auscultation led one to suppose. The cavity at the right apex was also smaller than one would have thought from auscultation signs. The cavities are well marked in the skiagram as two more or less round clear spaces surrounded by a dark shading. On looking at the lower parts of the lungs we see varying degrees of transparency mixed up with tracts of shading. This shading corresponds, no doubt, with consolidated lung tissue, but partly perhaps. with pleural adhesions.

I have quite lately had the opportunity of taking a skiagram of a pleural effusion (serum). It shows a uniform darkening of the whole of the affected side, quite unlike the mottling of the lung in tuberculosis. This darkening stands out in marked contrast to the transparent lung tissue on the unaffected side. The diaphragm also stands lower on the affected side. I think it is very probable that with the aid of the $\mathrm{x}$ rays one may be able to distinguish between serum 
F1G. 1.

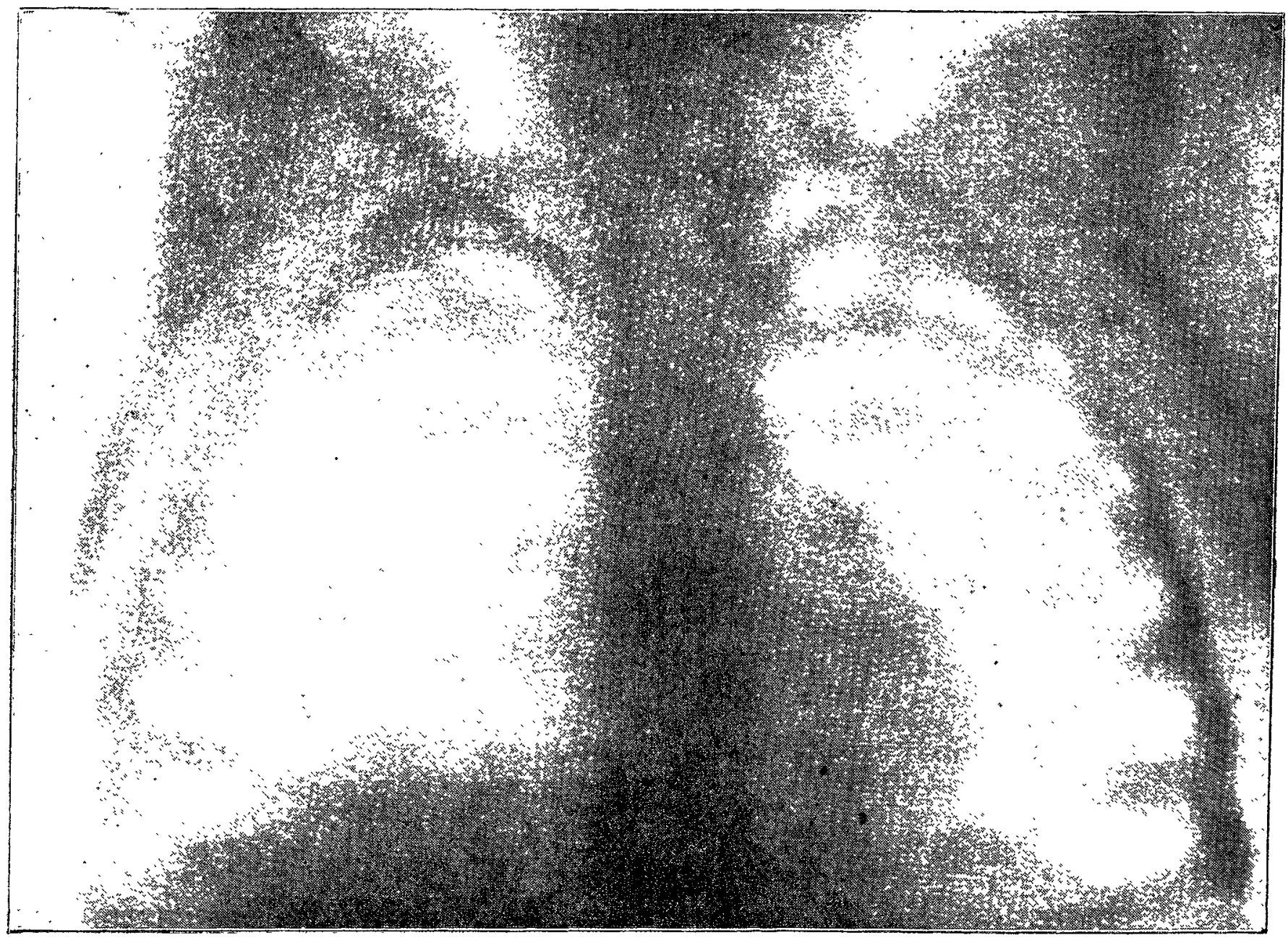

Skiagram it nommal chent.

FIC. 2.

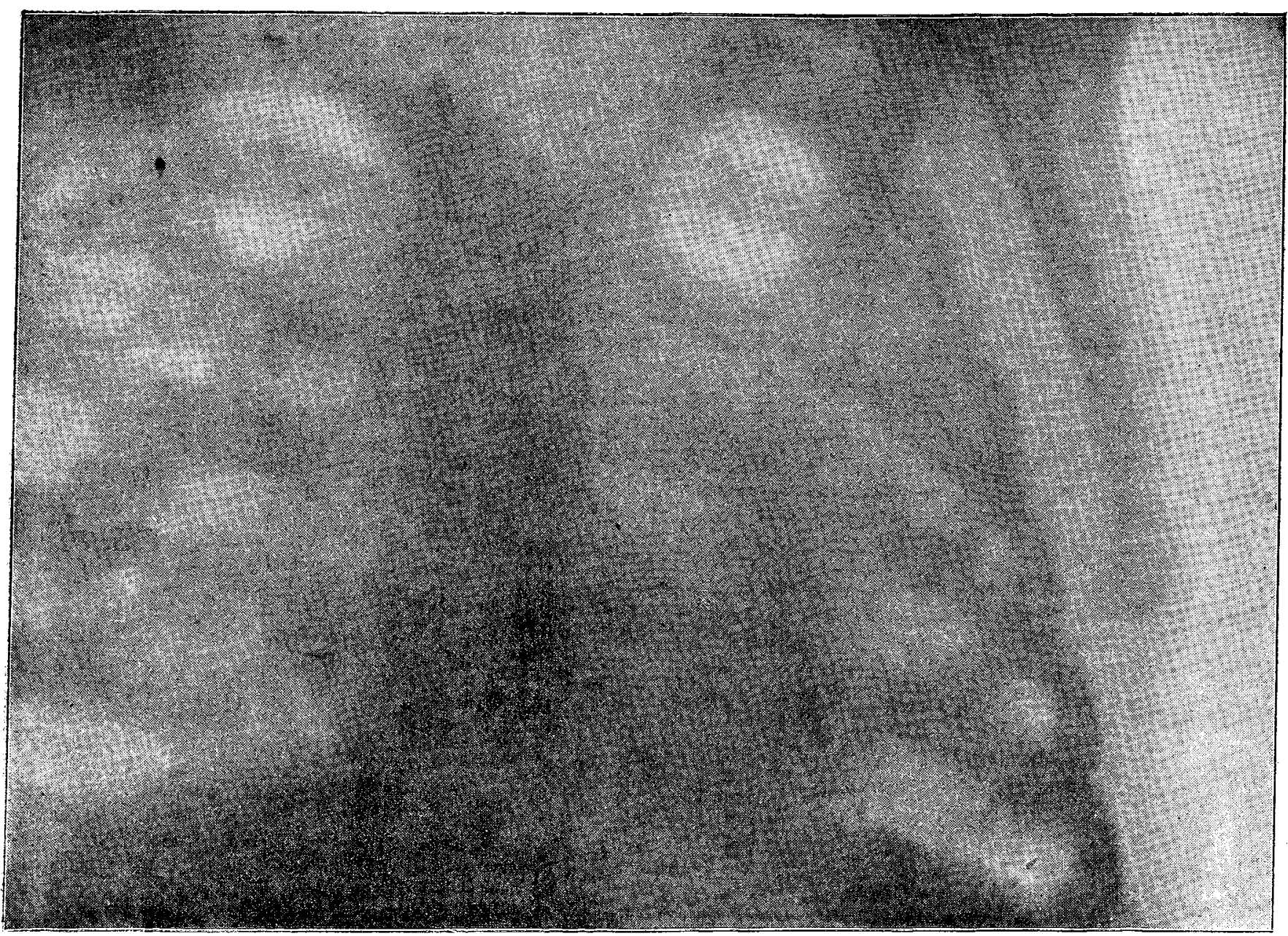

Skitgram of chest of chronic pulmonary tulerculosis, showing cavities at both apices. 


and pus, but at present $I$ have not had an opportunity of
making the observation. I think it is doubtful if the rays can enable one to distinguish between consolidation of the lower lobe of a lung and an effusion into the pleura.

Tarly cases of tuberculous consolidation of one or other apex display its presence by a dark shading at that apex, probably before physical signs become apparent. Enlarged bronchial glands, especially in children, are, according to Professor Bouchard and Professor Claude, easily distinguishable by the $\mathrm{x}$ rays, but of this $\mathrm{I}$ have had no experience. It does not appear likely that the $x$ rays will be of much use in the examination of the heart. True, they will show enlargement of the organ, but of this we have abundant proof without their aid. I think it is in lung disease, especially in tubercle of the lung, that they will be found of great ase both in diagnosis and prognosis. Both the accompanying skiagrams were taken by myself in the electrical department of St. Bartholomew's Hospital.

Harley-street, W.

\section{NASAL OBSTRUCTION AND EAR AFFECTIONS.}

BY MAYO COLLIER, M.S. LoND., F.R.C.S. ENG., SENIOR ASSISTANT SURGEON TO THE NORTHI-WEST LONDON HOSPITAL.

THE admitted difficulty in treating affections of the middle ear associated with deafness, tinnitus, or chronic discharges and the unsatisfactory results which follow in many cases the usual methods of treatment are due in a large measure to an improper understanding of the relationship and bearings of nasal obstruction as a primary cause or starting-point of most aural affections. If this be so it naturally follows, seeing that the study of aural diseases and their treatment dates back to a period long before a nasal speculum was dreamt of and long before a line of literature recorded a single observation or examination of the nose, that a minute and careful study of affections of the nasal chamber is a necessary preliminary to a proper understanding of most of the commoner affections of the ear.

"Throat deafness" is a well-recognised and significant term used and understood by professional and non-professional persons alike to indicate a state of things which may be literally interpreted as Eustachian obstruction the result of catarrh and swelling of the orifice of the Eustachian tube, usually a sequela or extension of nasal catarrh and consequently of nasal obstruction. Simple obstruction of the orifice of one or both Eustachian tubes following nasal catarrh is the starting-point or antecedent of a very large percentage of the commoner affections of the ear which make themselves apparent to the patient, as deafness, noises in the head, and "running" from the ear.

It is perfectly obvious that if the antecedent condition of Eustachian obstruction and catarrh were effectively treated and its cause was removed the more lasting and grave affections of the middle ear would be prevented and their subsequent treatment rendered unnecessary. I will enlarge my proposition and go one step further. Given chronic and wellmarked affections of the middle ear set up and induced by an original Eustachian catarrh and obstruction no permanent improvement can be hoped for or attained unless the fons et origo mali, the Eustachian obstruction, and its causa causans, the nasal obstruction, be removed.

I never find chronic deafness with or without noises in the head and discharges from the ear unassociated with nasal obstruction and I seldom have failed even in the most chronic and intractable cases to attain some degree of improvement either in the deafness, tinnitus, or discharge by removing the cause of the Eustachian obstruction--i.e., by ventilating the nose. To some persons it may not be apparent how an obstructed nostril can cause Eustachian obstruction and catarrh.

During inspiration the free ingress of air is requisite to fill the chest cavity on expansion of the thorax. An obstructed ingress means rarefaction of the air in the chest cavity and the passages leading thereto. During impeded nasal respiration the air in the post-nasal space is consequently of lesser clensity than the outside air. As a result of this the intravascular and extravascular pressures are not equilibrated (as in dry cupping) and swelling of the parts with engorgement. of the vessels takes place. This vascular engorgement and consequent swelling of the orifice of the Eustachian tubes involves obstruction to the free passage or ingress of air to the tympanic cavity as well as often an extension of the catarrhal process through the Eustachian canal to the tympanic cavity.

To follow this apparently simple catarrh a step or two further, the fact of obstructing the free inlet of air to the tympanic cavity causes a serious physical disturbance. The air present in the tympanic cavity becomes shortly absorbed, causing a negative intra-tympanic pressure. This causes engorgement of the whole lining memabrane of the tympanum, with possible and probable effusion into that cavity according to the degree of the vacuum. Added to this the unsupported drum-head is driven inwards by the unbalanced atmospheric pressure and deafness with or without tinnitus is the probable result. The sequence of trouble is not yet complete. The extension of the catarrhal process to the tymnanic cavity may mean the introduction of certain pathogenic organisms and the consequent more acute state known as abscess of the middle ear.

The formation of pus is usually associated with pain in the ear, severe in character; high temperature with or without delirium ; and sooner or later bursting of the drumhead with discharge of pus from the external ear.

The vista of possible consequences of our original Eustachian obstruction from catarrh is not yet obscured. The immediate result of an acute abscess of the middle ear is usually perforation of the drum-head with cessation of the acute symptoms and relief to the patient. Not so always with infants and children, convulsions and death are a common termination of the acute stage.

The after-stage of a tympanic abscess may be short or long. Healing of the perforation may take place, to re-open with the next cold in the nose and throat and again discharge, lapsing ultimately into a chronic ear discharge. The chronic ear discharge may, but rarely does, end in spontaneous cure. More often a chronic discharge from the ear is kept up for many years and either ends in death from abscess of the brain or septicœmia from infection of the lateral sinus, but more often in caries of the petrous bone and impairment of the general health and death from some indirect cause.

In drawing this sketch of a simple cold in the nose leading to throat and post-nasal catarrh and the consequent Eustachian obstruction I have not exaggerated in the smallest degree. My object is to impress upon those who have the care and the treatment of these cases that simple and appropriate treatment applied to the original catarrh and obstruction would prevent and save this long sequence of aural troubles. An astringent and antiseptic wash to the nose would restore nasal respiration, relieve Eustachian congestion and obstruction, and permit a free ingress of air to the tympanic cavity. In chronic cases of deafness with tinnitus and discharge due originally to Eustachian catarrh and obstruction the same treatment applies, and is absolutely essential and a sine quâ non of any permanent improvement and success in these cases. A wash followed by the galvano-cautery or other methods of ventilating the nose is the first step in treating these chronic aural affections. Then and then only will the Politzer bag and the Eustachian catheter avail anything. Harley-street, $w$.

Gift of a WATER-supply.-Mr. D. R. Scratton on Oct. 6th presented a water-supply to the village of East Ogwell, near Newton Abbot, Deron. The cost of the gift is about $£ 3000$. Mrs. Scratton turned on the water with a golden key presented by the villagers. The day was observed as a general holiday and Mr. Scratton was presented with an illuminated address of thanks.

Clinical Society of Manchester.-At the annual meeting of this society, held on Oct. 4th, the following office-bearers were elected for the session 1898-99:President: Dr. A. Hill Griffith. Vice-presidents : Dr. E. S. Reynolds and Mr. E. Roberts. Treasurer: Dr. C. H. Braddon. Secretary: Dr. T. A. Helme. Librarian : Mr. J. W. Smith. Auditors: Mr. C. F. H. Kitchen and Dr. T. Porter. Committee : Dr. Alfred Brown, Dr. R. Crean, Dr. J. Daniel, Dr. J. W. Hamill, Dr. T. Harris, Dr. J. Holmes, Dr. T. N. Kelynack, Dr. M. P. Ledward, Mr. H. Lund, Mr. C. T. B. Maisey, Dr. E. T. Milner. Dr. R. T. Parkinson, Mr. J. E. Platt, Mr. J. Rust, and Mr. F. H. Westmacott. 